\title{
Multi-wavelength study of the eclipsing polar UZ Fornacis*
}

\section{Zwidofhelangani N. Khangale ${ }^{\dagger}$}

South African Astronomical Observatory, Observatory Road, Observatory, 7925, Cape Town, RSA

Department of Astronomy, University of Cape Town, Private Bag X1, Rondebosch 7701, South Africa

E-mail: khangaleznesaao.ac.za

\section{Stephen B. Potter}

South African Astronomical Observatory, Observatory Road, Observatory, 7925, Cape Town, RSA

E-mail: sbpesaao.ac.za

\section{Patrick A. Woudt}

Department of Astronomy, University of Cape Town, Private Bag X1, Rondebosch 7701, South Africa

E-mail: pwoudteast.uct.ac.za

\begin{abstract}
We present phase-resolved spectroscopy and circular spectropolarimetry of the eclipsing polar UZ Fornacis. Doppler tomography of the strongest features $(\mathrm{H} \beta$ line) using the inside-out technique revealed the presence of three emission regions: from the irradiated face of the secondary star, the ballistic stream and the threading region, and the magnetic confined accretion stream. The total intensity spectrum shows broad emission features that span the entire bandwidths with the continuum that rises in the blue. The circularly polarized spectrum shows the presence of three cyclotron harmonics at $4500 \AA, 6000 \AA$ and $7700 \AA$, corresponding to harmonic numbers 4,3 , and 2 , respectively. These features are dominant before the eclipse and disappears after the eclipse. The harmonics are consistent with the magnetic field strength of $\sim 57 \mathrm{MG}$. We also present phaseresolved circular and linear photopolarimetry to complement the spectro-polarimetry around the times of eclipse. Our current MeerKAT observations show a faint source which has a peak flux density of $31 \pm 6 \mu \mathrm{Jy}$ per beam at a position of UZ For. We place the formal upper limit of $\sim 37$ $\mu \mathrm{Jy}$ per beam at $1.28 \mathrm{GHz}$.
\end{abstract}

Palermo Workshop 2019: The Golden Age of Cataclysmic Variables and Related Objects - V 2-7 September 2019

Splendid Hotel La Torre, Mondello, Palermo, Italy

\footnotetext{
*Based on observations made with the Southern African Large Telescope (SALT).

${ }^{\dagger}$ Speaker.
} 


\section{Introduction}

The AM Herculis systems, or polars, are a sub-class of magnetic cataclysmic variable (mCV) stars consisting of a strongly magnetized white dwarf primary $(B \approx 10-230 \mathrm{MG}$, e.g. Schmidt et al. 1996) and a low-mass main-sequence secondary star. The interaction between the magnetic field of the white dwarf (WD) and that of the secondary star results in synchronous rotation of the two stars. The presence of the strong magnetic field in the WD prevents the formation of an accretion disc. The red dwarf is constantly transferring material to the WD via Roche lobe overflow. The material from the WD follow a ballistic stream trajectory from the inner Lagrangian point $\left(L_{1}\right)$ and accelerates to supersonic speeds towards the WD until, at some distance from the WD, the magnetic pressure overwhelms the ram pressure of the ballistic stream. At this point, the ballistic stream is diverted from the orbital plane of the binary and follows a trajectory along the magnetic field lines of the WD. The material in the magnetic stream is ionized due to collisions within the stream and also by X-rays from the accretion region on the surface of the WD. At some height above the WD surface, a stand-off shock is formed when the supersonic free-falling material become subsonic. The typical temperature of the material in the shock reaches $10-50 \mathrm{keV}$ and this result in the gas being highly ionized. The heated plasma cools down as it settles onto the surface of the WD resulting in a stratified post-shock region. The high density and temperatures (thermal velocities) in the post-shock region force the electrons to gyrate around the magnetic field lines emitting cyclotron radiation which is highly polarized. The AM Her types are recognized for their high degree of polarization (e.g. AM Her Tapia 1977). The post-shock region is also responsible for the emission of X-ray bremsstrahlung radiation and most of this is reprocessed by the surface of the WD and re-emitted as soft X-rays. Accretion onto the WD occurs over a small area on the surface of the WD near one or both the magnetic poles. For review on polars see, for example, Cropper (1990) or Warner (1995). In this proceeding we present photometry, spectroscopy and circular spectropolarimetry as well as radio observations of the eclipsing polar UZ Fornacis (hereafter UZ For).

\section{Observations}

Spectroscopic observations of UZ For were the made with Southern African Large Telescope (SALT, Buckley et al. 2006), situated at the site of the South African Astronomical Observatory (SAAO) in Sutherland, over five nights between 2013 January 03 and 2014 January 30 using the Robert Stobbie Spectrograph (RSS; Burgh et al. 2003; Kobulnicky et al. 2003) in long-slit mode using the slit width of $1.5^{\prime \prime}$. Data reduction was carried out using the PYSALT software package (Crawford et al., 2010) and IRAF reduction procedures. Relative flux correction of the blue and red spectra was based on the sensitivity function of the spectro-photometric standard stars LTT 4364 and EG21 (Baldwin \& Stone, 1984), respectively.

Photopolarimetry observations of UZ For were made on the night of 2018 October 4 with the SAAO 1.9-m telescope using the HIgh-speed Photo-POlarimeter (HIPPO, Potter et al. 2010). The HIPPO instrument was operated in its simultaneous linear and circular polarimetry and photopolarimetry mode (all-Stokes). The observations were clear filtered using the RCA31034A GaAs 
photomultiplier tubes. Data reduction was carried out following the procedures described in Potter et al. (2010).

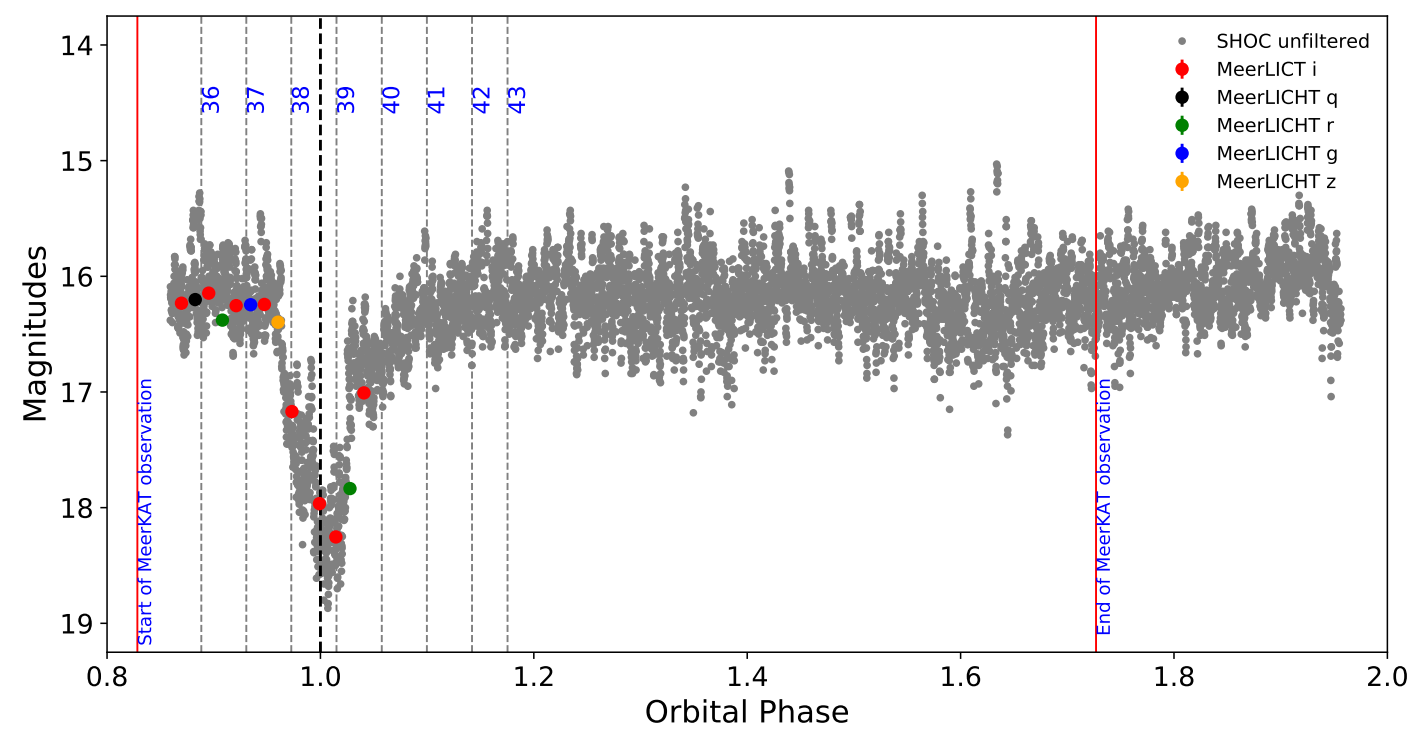

Figure 1: Simultaneous eclipse light curve of UZ For obtained with SHOC (grey dots) and MeerLICHT (circles) on 2018 November 6 . The red vertical lines marks the start- and end-time of the MeerKAT observations. The vertical grey lines mark the mid-exposure of the eight individual spectropolarimetry exposures taken with SALT whereas the black dashed line represent the time of mid-eclipse.

We obtained coordinated simultaneous photometry (SHOC and MeerLICHT), spectropolarimetry with SALT and MeerKAT radio observations of UZ For on 2018 November 6. This was the first time such observations were conducted at SAAO. The high-speed photometric observations were obtained using the SAAO 1.9-m telescope that is equipped with the Sutherland High-speed Optical Camera (SHOC, Coppejans et al. 2013) and conditions were photometric. The SHOC detector was used in frame-transfer mode with a clear filter and binning of $8 \times 8$ was used. A cadence of one second was used, and the resulting data cubes were reduced using the SHOC pipeline that is described in Coppejans et al. (2013).

Further photometric data was obtained using the MeerLICHT (Bloemen et al., 2016) telescope utilizing the five filters, e.g. $i, q, r, g$ and $z$. The MeerLICHT telescope is a fully robotic $0.65-\mathrm{m}$ telescope with an instantaneous field of view matching that of MeerKAT (2.7 square degrees). MeerLICHT is equipped with an STA1600 detector which provides a 2.7 square degree field of view at $0.56 \mathrm{arcsec} / \mathrm{pixel}$. The observations lasted for about 30 minutes and covers most of the primary eclipse.

Spectropolarimetry observations of UZ For was obtained with the SALT RSS instrument in circular spectropolarimetry (Nordsieck et al., 2003) mode. A total of eight exposures of $300 \mathrm{~s} \mathrm{each,}$ with phase resolution of about 0.05 of the orbital period and spectral resolution of $4 \AA$, containing the ordinary $(\mathrm{O})$ and extraordinary (E) beams were obtained around the eclipse (see Fig. 1). An exposure of Argon lamp was taken after the science frames for wavelength calibration purposes. The observations of the spectrophotometric standard star (HILT600, unpolarized optical calibrator) 
were obtained on the night of 2018 December 4 with the same setup as our science exposures. The CCD pre-processing of the observations was performed using the polsalt-beta ${ }^{1}$ software (Potter et al., 2016) based on PYSALT package, this includes overscan correction, bias subtraction and gain correction. The wavelength calibration for both the $\mathrm{O}$ and $\mathrm{E}$ beams was performed using the Argon lamps taken with the same observation septup. We computed the degree of circular polarization $(V / I)$ from two consecutive exposures, with the quarter wave retarder plate rotated by $\pm 45^{\circ}$, using Equ. 2.1 below (adopted from Euchner et al. 2005):

$$
\frac{V}{I}=\frac{1}{2}\left[\left(\frac{f^{\mathrm{o}}-f^{\mathrm{e}}}{f^{\mathrm{o}}+f^{\mathrm{e}}}\right)_{315^{\circ}}-\left(\frac{f^{\mathrm{o}}-f^{\mathrm{e}}}{f^{\mathrm{o}}+f^{\mathrm{e}}}\right)_{45^{\circ}}\right]
$$

where $45^{\circ}$ and $315^{\circ}$ indicate the position angle of the quarter wave plate and $f^{\circ}$ and $f^{\mathrm{e}}$ are the ordinary and extraordinary beams, respectively. The total relative intensity was obtained by adding the sum of the $\mathrm{O}$ and $\mathrm{E}$ beams. For relative flux calibration we used the spectrophotometric standard star HILT600.

Radio observations of UZ For and the surrounding field were obtained on 2018 November 6 using the MeerKAT radio telescope (Camilo et al., 2018; Jonas \& MeerKAT Team, 2016) at the site of the South African Radio Astronomical Observatory (SARAO), located in the Karoo desert in South Africa. These observations are part of the ThunderKAT Large Survey Project (Fender et al., 2017). The observations were taken using 62 of the MeerKAT antennas, at a central frequency of $1.28 \mathrm{GHz}$, with a total bandwidth of $856 \mathrm{MHz}$ split into 4096 channels. Visibilities were recorded every 8 seconds. The band-pass and flux calibrator, PKS J0408-6545, was observed for 10 minutes at the beginning of the observation. Thereafter the gain calibrator, PKS J0409-1757, and UZ For were observed, for approximately 1.5 minutes and 15 minutes respectively, alternating between them repeatedly for the remainder of the observation. The total integration time on UZ For was approximately 100 minutes. We used AOFlagger (Offringa et al., 2012) to flag the data (i.e. removing RFI) and then the raw data were binned into 8 channels per bin, resulting in 512 channels each with a bandwidth of $1.67 \mathrm{MHz}$. Data reduction and first generation calibration were executed using standard procedures in CASA (McMullin et al., 2007). We made use of the facet based radio-imaging package DDFacet (Tasse et al., 2018) for imaging, implementing the SSDClean deconvolution algorithm and Briggs weighting with a robust parameter of 0.0. No self-calibration was implemented. Fitting was done in the image domain using the IMFIT task in CASA and noise levels were measured in the vicinity of the expected position of the source.

We calculated the phases using the ephemeris listed in Khangale et al. (2019). We corrected all the times for the light travel-time to the barycentre of the solar system (i.e. converted to the barycentric dynamical times (TDB) as Barycentric Julian Dates (BJD; Eastman et al. 2010)).

\section{Results}

\subsection{Photometry}

Figure 1 shows the light curve of UZ For obtained with the SHOC instrument. MeerLICHT magnitudes are also shown in the figure and trace the primary eclipse. The magnitudes variation

\footnotetext{
${ }^{1}$ For more details see https://github. com/saltastro/polsalt/.
} 
from SHOC and HIPPO are consistent with each other. The light curve shows lot of flickering when the system is out of eclipse. The shape of the eclipse is similar to those recorded in the literature (e.g. Bailey \& Cropper 1991; Khangale et al. 2019) and the out-of-eclipse shape of the light curve is similar to that of Perryman et al. (2001).

\subsection{Spectroscopy and Doppler tomography}
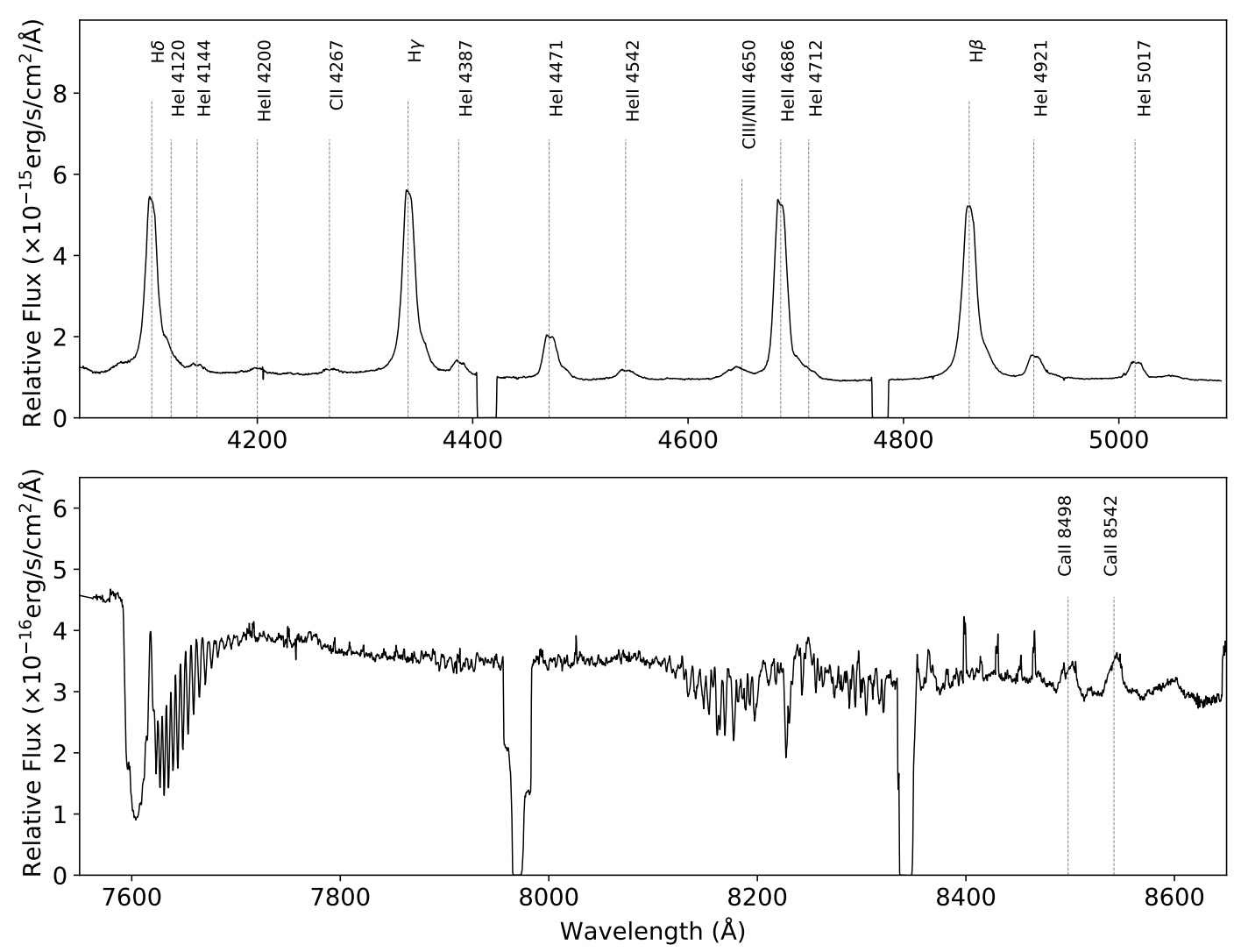

Figure 2: Averaged wavelength calibrated blue (top) and red (bottom) spectra of UZ For obtained with SALT. Prominent emission and absorption features have been labeled.

Figure 2 (top panel) shows the averaged blue spectrum of UZ For taken over three nights. The blue spectrum shows the presence of single- and/or double-peaked emission from the Balmer lines, HeII lines and HeI lines. HeII $4686 \AA$ and the Balmer lines dominate the continuum. The Bowen

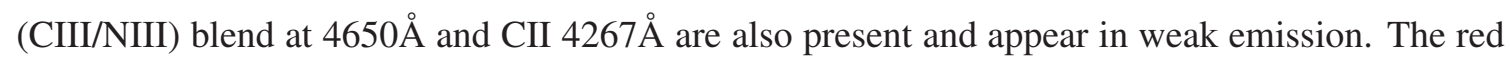
spectrum of UZ For, shown in the bottom panel of Fig. 2, shows a continuum dominated by telluric lines in absorption. The features at $7600 \AA$ are due to absorption by the Earth's atmosphere. There is strong emission from the CaII lines at $8498 \AA$ and $8542 \AA$.

We used the strongest features from the blue spectra to compute Doppler maps of emission lines for further investigation utilizing the Doppler tomography code, described in Kotze et al. (2015). In Figs 3 and 4, we present both the standard and inside-out Doppler maps and trailed spectra of UZ For using the $\mathrm{H} \beta$ line. We have over-plotted a model with WD mass, $M_{1}=0.71$ 


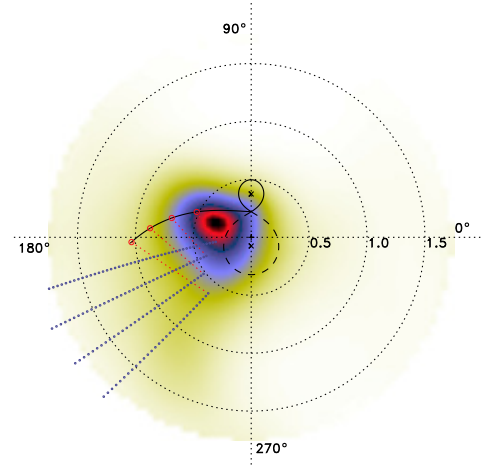

$(\mathrm{v}, \theta)\left[10^{3} \mathrm{~km} \mathrm{~s}^{-1}\right.$, degrees $]$

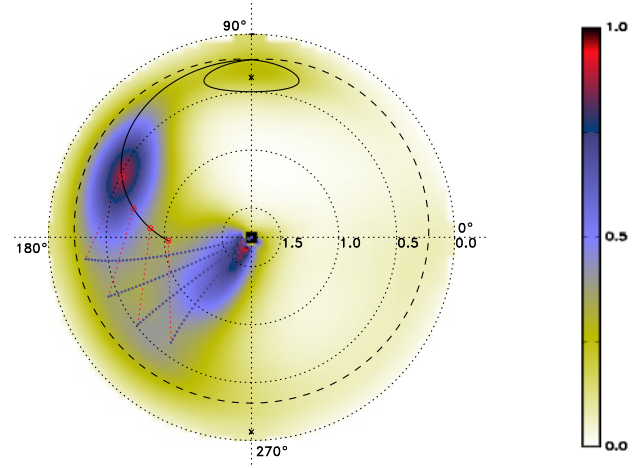

$(v, \theta)\left[10^{3} \mathrm{~km} \mathrm{~s}^{-1}\right.$, degrees $]$
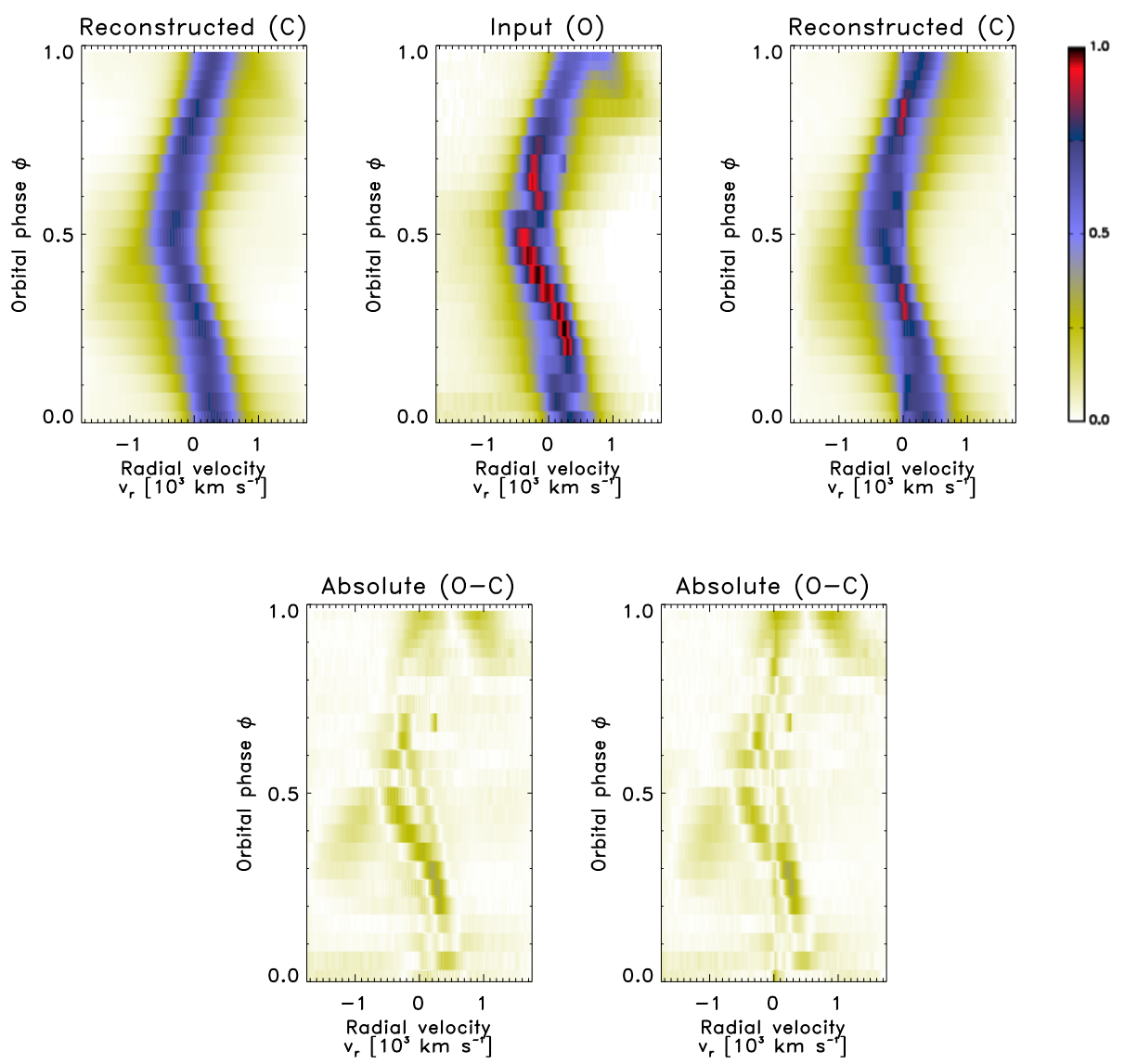

Figure 3: Standard and inside-out Doppler maps and trailed observed and reconstructed spectra of UZ For using the $\mathrm{H} \beta$ line. Top row: the standard (left) and inside-out (right) Doppler tomograms. Second row: the input trailed spectra (centre) with the reconstructed trailed spectra for the standard (left) and inside-out (right) tomograms, respectively. The bottom row shows corresponding trailed residual spectra. 
$M_{\odot}$, mass ratio $\left(q=\frac{M_{2}}{M_{1}}\right)$ of 0.2 and inclination, $i=81^{\circ}$ (Bailey \& Cropper, 1991). The Roche lobe of the WD is shown with a dashed line whereas that of the secondary is shown with a solid line in both the standard and inside-out Doppler maps. The trajectory of the ballistic stream is marked with a solid line from $L_{1}$ up to $45^{\circ}$ in azimuth around the primary. The magnetic dipole trajectories are marked with thin dotted blue lines and are calculated at $10^{\circ}$ intervals in azimuth around the primary. The colour-bars in both figures, to the right of the tomograms, show the scale with which the Doppler maps and trailed spectra were produced. The bottom rows in each figure shows the input-minus-reconstructed $(\mathrm{O}-\mathrm{C})$ residual spectra of the same line based on the standard and inside-out reconstructed trailed spectra. The colour bars to the right of the Doppler maps and trailed spectra show the scale with which the amplitude maps are presented. We start by discussing the input or observed (O) trailed spectra (Fig. 3, second row centre) since it is common amongst the two methods followed by the Doppler maps.

The trailed spectra reveals the presence of three distinct emission components. The first is a relatively narrow component (red color) which is brightest around phase 0.2 to 0.5 (and possibly phase $0.55-0.75$ ). This component has a low-velocity amplitude and is understood to be associated with the irradiated face of the secondary star. The second component is a broad emission line (blue color) which has a high velocity amplitude. This component crosses zero velocity from red to blue at around phase 0.3 and then from blue to red at phase around 0.8. It has a maximum red-shift at phase 0.95 and a maximum blue-shift at phase 0.5 . The low- and mid-velocity components are blended together after phase 0.3. The third component is a relatively broad feature (yellow color) which is visible throughout the orbital phase. It is blue-shifted around phases $0.0-0.5$ and redshifted around phases 0.5-0.95. The two broad emission features, blue and yellow, are associated with emission produced in different parts of the accretion flow.

\subsubsection{Standard and inside-out Doppler tomograms}

The reconstructed or calculated (C) trailed spectra based on the standard and inside-out methods reproduce the basic structure of the observed trail spectra. The low-velocity (red color) component is absent in the reconstructed trailed spectra. The observed flux distribution is not reproduced in both the standard and inside-out trailed reconstructed spectra. The trailed residual spectra (Fig. 3 , bottom panels) show the difference between the observed and reconstructed spectra. There is no difference between the trailed residual spectra based on standard method compared to the one based on inside-out method. However, there is some residual structures remain when subtracting the reconstructed trailed spectra from the observed trailed spectra. The residuals has an rms of 0.223 and 0.221 for standard and inside-out method, respectively.

Figure 3 (top row) shows the standard (left) and inside-out (right) Doppler tomogram of the $\mathrm{H} \beta$ line. It is clear from the figure that emission from the ballistic stream and threading region dominates the tomograms irrespective of the method used. There is little to no evidence of emission from the vicinity of the secondary star in the standard tomogram. But emission from the secondary is clearly seen in the inside-out tomogram, where the three components seen in the observed trailed spectra are projected in various parts of the tomogram. In the inside-out tomogram, the secondary star which is seen as a diffuse feature covering the Roche lobe of the secondary star. The magnetic confined stream is also revealed in both the tomograms but is more pronounced in the inside- 
out method. Overall, more features are seen in the inside-out tomograms than in the standard tomograms.

\subsubsection{Standard and inside-out modulation amplitude maps}

The top panels of Fig. 4 show the flux modulation amplitude Doppler maps of the $H \beta$ line in order to map out emissions which vary as a function of the orbital phase. The flux modulation Doppler maps presented here are based on ten consecutive half-phases of $\mathrm{H} \beta$ (i.e., $0.0-0.5,0.2-0.6$, $\ldots, 0.7-0.2$, etc). Also presented are the trailed spectra (second row) and residual spectra (bottom row).

The trailed reconstructed spectra (Fig. 4, second row) for both the standard and inside-out method, based on the summed half-phases, show the mid- and high-velocity component throughout the orbital phase. The low-velocity component is visible at some orbital phase. With the modulation technique, the basic structure of the observed trailed spectra is reproduced. In addition to that, the patches of the low-velocity component is seen in the reconstructed trailed spectra. However, the reconstructed trailed spectra based on the inside-out method reproduces the observed trailed spectra better. Once again, there is no big difference between the residuals spectra from both methods, their rms were similar.

The standard modulated amplitude Doppler map, Fig. 4 top left panel, reveals that the ballistic and magnetic confined stream are the most flux modulated components. The emission is concentrated in the vicinity of the threading region (and the ballistic stream) and some emission is seen forming a tail along the magnetic field lines. However, it is not clear if the secondary star is also shown to modulate. The inside-out modulation amplitude map, Fig. 4 top right panel, shows that the ballistic and magnetic confined stream are the most flux modulated components. In addition to these two components, the secondary star is also shown to be flux modulated. This is indicated by a dense yellow patch overlaid on the Roche lobe of the secondary star. The modulation of the secondary star is more clear in the inside-out Doppler map.

\subsection{Photopolarimetry}

Our simultaneous linear and circular polarization results are shown in Fig. 5. The top panel shows photometric light curve from HIPPO with a duration of 1.53 hours. The shape of the eclipse is similar to that shown in Fig. 1 with clear defined ingress and egress of the main accretion spot. The out-of-eclipse variability is consistent with low amplitude flickering than in Fig. 1. The clear-filtered circular polarimetric observations (Fig. 5, middle panel) show polarization variability between 0 and $-5 \%$. The out-of-eclipse circular polarization between phases 0.5 and 0.7 is consistent with zero. Before the eclipse, from phase $0.7-0.95$, the polarization increases to $-5 \%$. This is because the region emitting cyclotron radiation is visible to the observer around these phases. During the eclipse, the total flux decreases resulting in large error-bars for polarization. After the eclipse, from phases 1.03-1.07, the emission is still negatively polarized with polarization ranging from 0 to $-5 \%$. After phase 1.07, UZ For shows a mixture of polarization which are consistent with zero. The bottom panel of Fig. 5 shows the percentage of linear polarization. The clear-filtered linear polarimetry shows variability between 0 and $10 \%$. The out-of-eclipse linear polarization, from phase $0.5-0.7$, is less than 5\%. Before the eclipse, phases $0.7-0.95$, the level polarization increases by a few percent $(<10 \%)-$ consistent with the circular polarization. During the eclipse, the 


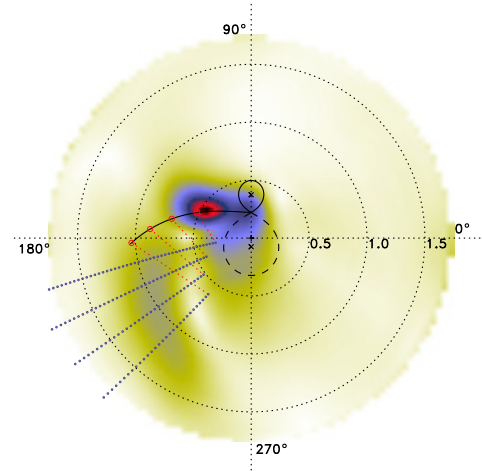

$(\mathrm{v}, \theta)\left[10^{\mathrm{s}} \mathrm{km} \mathrm{s}^{-1}\right.$, degrees $]$

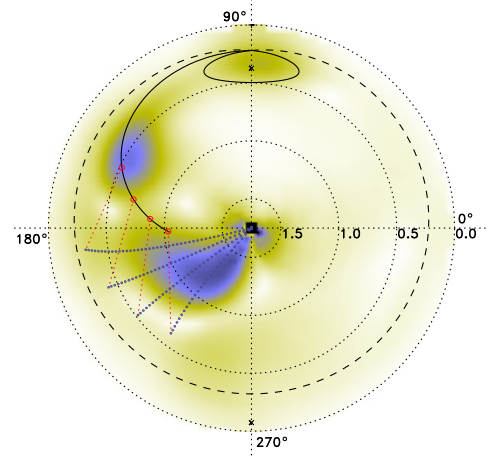

$(\mathrm{v}, \theta)\left[10^{3} \mathrm{~km} \mathrm{~s}^{-1}\right.$, degrees $]$
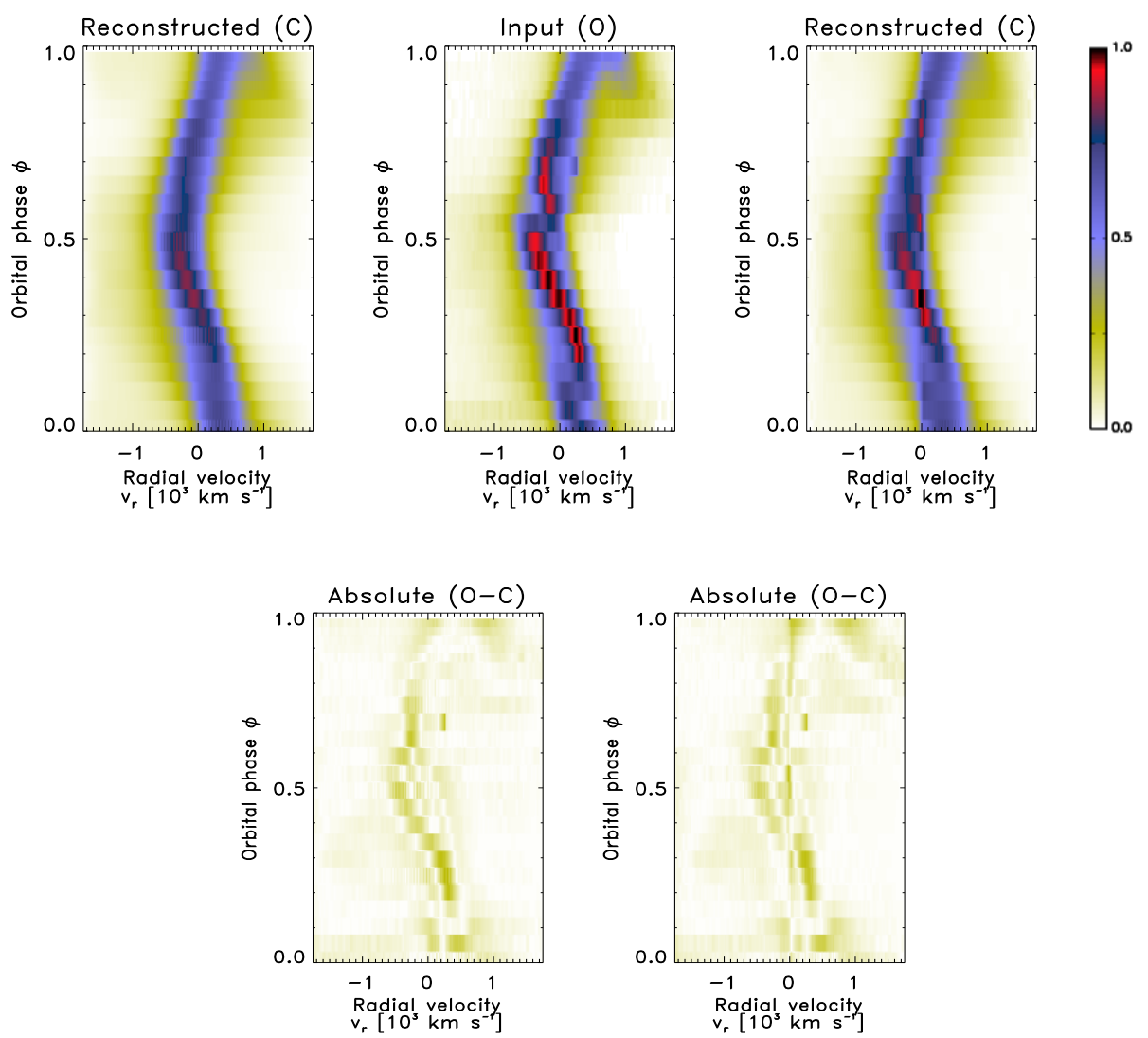

Figure 4: Standard and inside-out Doppler maps and trailed observed and reconstructed spectra of UZ For using the $H \beta$ line. Top row: the standard and inside-out modulation amplitude flux Doppler maps. Second row: the input trailed spectra (centre) with the summed reconstructed trailed spectra for the ten consecutive half-phases for standard (left) and inside-out (right), respectively. The bottom row shows the corresponding trailed summed absolute residual spectra. 


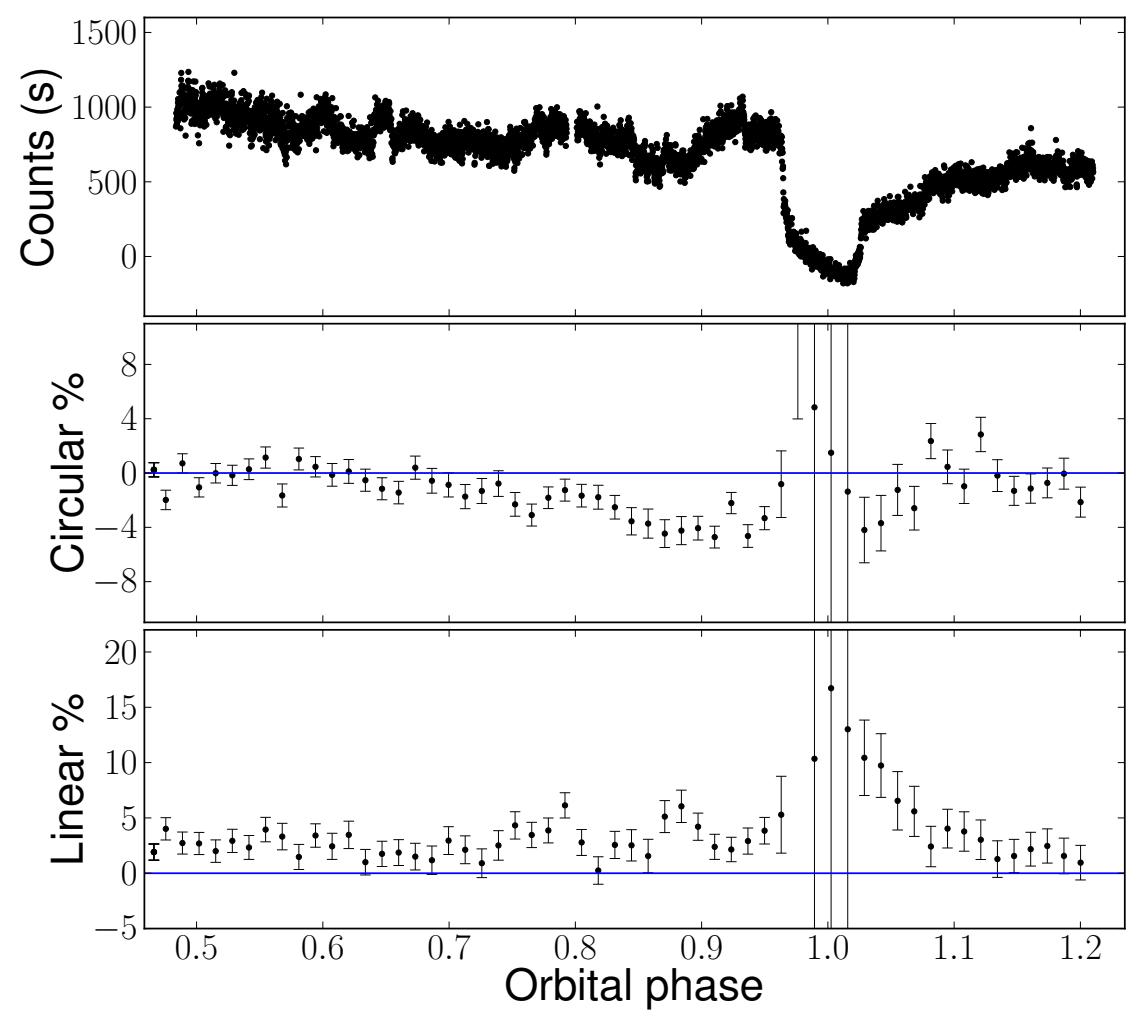

Figure 5: Photopolarimetry from 2018 October 4 made with the HIPPO instrument. Top to bottom panels correspond to photometry, percentage circular and linear polarization.

total flux decreases resulting in large error-bars for polarization. After the eclipse, there is a pulse of linear polarization reaching about $10 \%$ and decreasing gradually before flattening out between phase 1.1-1.2 and beyond.

\subsection{Circular spectropolarimetry}

Figures 6(a) to 6(d) show the time-sequence of circular spectropolarimetry obtained before the eclipse, during the eclipse, emerging out of the eclipse and after the eclipse. Each panel of the figure (from top to bottom), shows the total flux spectra, the percentage of circular polarization and the total circularly polarized flux are shown. The total flux spectra show a continuum which rises in the blue and is dominated by broad emission features covering the entire waveband. The total flux spectra possibly show a broad hump around $5500 \AA$.

\subsubsection{Percentage circularly polarized spectra}

The middle panels of Figs 6(a) to 6(d) show the time-sequence of the percentage of circularly polarized spectra of UZ For. They show strong negative circular polarization (up to -8\%) in the blue and decreasing gradually towards the red. The grey dashed vertical lines mark the location of the emission lines (as seen in the top panels) and it is clear that there are humps at their locations since emission lines are not polarized. The circular polarization spectra show the presence of 


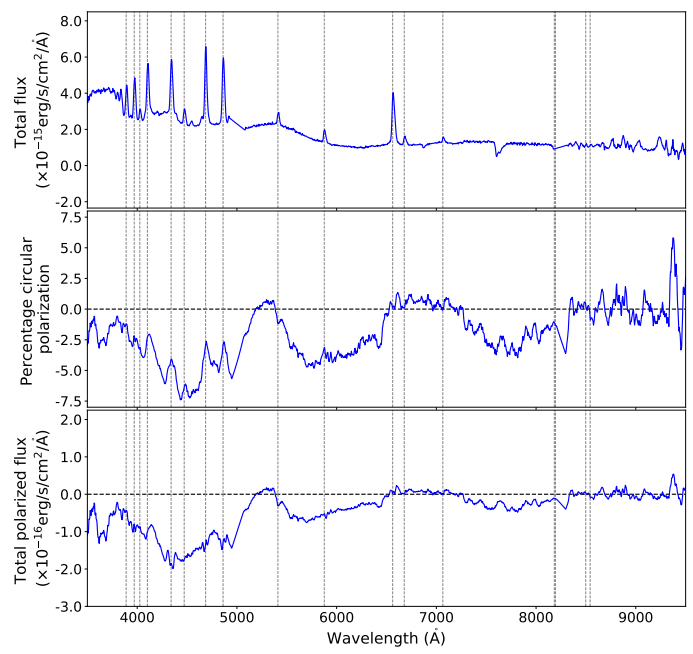

(a) Before the eclipse $(\phi=0.89-0.93)$

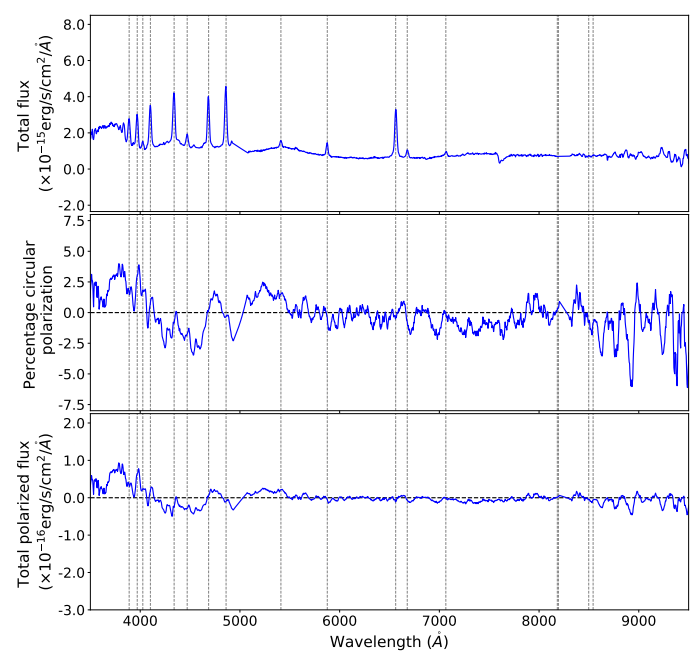

(c) Emerging out of eclipse $(\phi=1.06-1.10)$

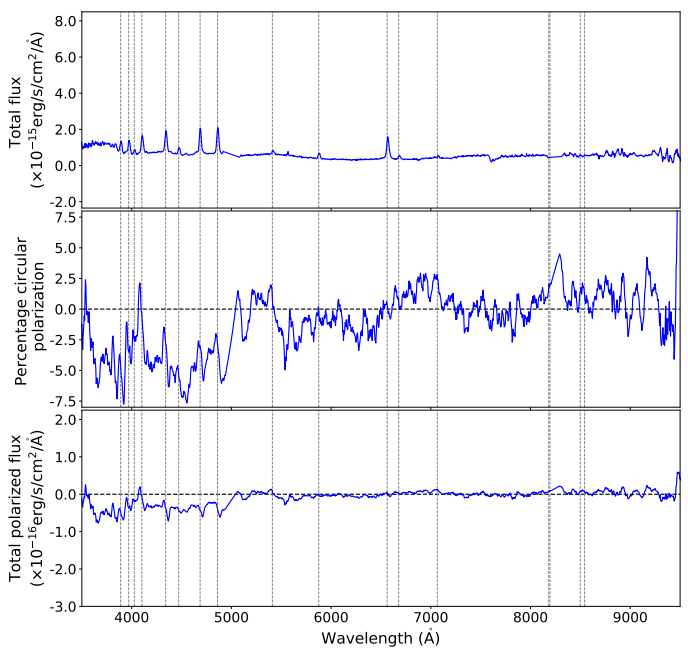

(b) During the eclipse $(\phi=0.97-1.02)$

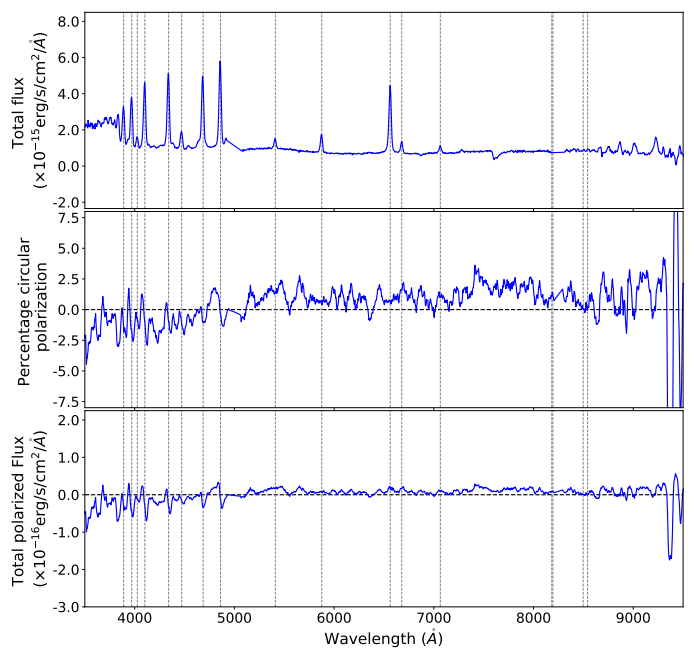

(d) After the eclipse $(\phi=1.14-1.18)$

Figure 6: Spectra showing cyclotron emission lines in total flux (top panel), circular polarization (middle panel) and the total polarized flux (bottom panel) of UZ For.

three dips, centred at $\sim 4500,6000$ and $7800 \AA$, that are understood as cyclotron harmonics due to cyclotron emission from a hot plasma. The harmonics are more visible in Fig. 6(a) (middle panel) before the eclipse. During the eclipse, Fig. 6(b) middle panel, the strength of the harmonics are significantly reduced, especially at longer wavelengths. The reason we see the harmonics during the eclipse is due to that the first exposure started before the ingress time and the second exposure was taken during mid-eclipse. When the system is emerging out of the eclipse, Fig. 6(c), there is some polarization in the blue part of the spectra. After the eclipse (Fig. 6(d) middle panel) over most of the observed wavelength, the circularly polarized spectra are consistent with $0 \%$ circular polarization. But between 7000 and $9000 \AA$, there is a marginal detection of positive polarization. 


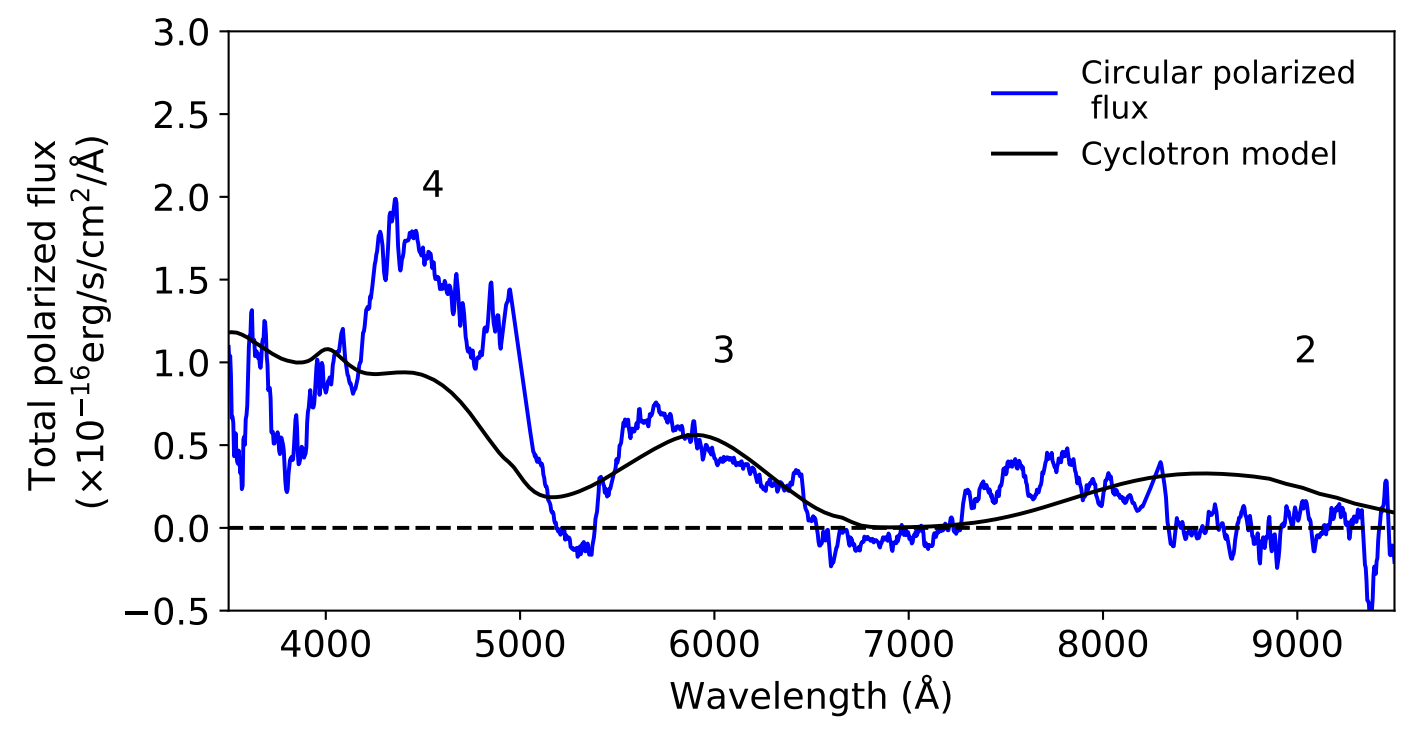

Figure 7: Total circularly polarized flux (blue) of UZ For and overlaid is the pure cyclotron model (black) with the magnetic field of $57 \mathrm{MG}$. The numbers 2, 3 and 4 marks the theoretical positions of the three harmonic features.

\subsubsection{Total circularly polarized flux}

We multiplied the total flux spectra by the percentage of circularly polarized spectra to get the total circularly polarized flux. The total polarized flux is of pure cyclotron origin and is free of contamination, e.g., emission from the secondary star. The results are shown in the bottom panels of Figs 6(a) to 6(d). As expected, much of the circularly polarized flux is seen in the blue in Fig. 6(a) (bottom panel) just before the eclipse and where the percentage of polarization reaches $\sim-8 \%$. During the eclipse (Fig. 6(b), bottom panel), some polarized flux is still seen in the blue end of the spectra. This implies that either the accretion spot emitting cyclotron radiation is not completely eclipsed during the primary eclipse or there is second region also emitting cyclotron radiation. After the eclipse, Figs 6(c) and 6(d), little or zero polarized flux is seen and the continuum is much flatter implying that the accretion spot emitting cyclotron radiation has moved away from the line of sight of the observer.

We modelled the circularly polarized flux at phase 0.91 (Fig. 6(a), bottom panel) since this shows broad features which peaks at $\sim 4500 \AA, 6000 \AA$ and $7800 \AA$, respectively. These features display the characteristic properties predicted by the theory for cyclotron emission from a hot plasma (Wickramasinghe \& Meggitt, 1982). We took the absolute value of polarization, and then modelled the positive total circularly polarized flux following the cyclotron emission models from the stratified accretion shocks of Potter (1998) as described Potter et al. (2002). The stratified accretion shocks model uses on hydrodynamic model of Cropper et al. (1999) to determine the structure of the post-shocks regions. The intensity and polarization of the emission are calculated using the 4-stokes formulation for cyclotron opacity and radiative transfer detailed in Meggitt \& Wickramasinghe (1982) and Wickramasinghe \& Meggitt (1985). The results are shown in Fig. 7 and we have over-plotted a pure cyclotron model with the magnetic field of 57 MG viewed at an 
angle of $70^{\circ}$ to the line of sight. The three cyclotron features mentioned above may be identified with harmonic numbers 4,3 and 2 .

We also used Equ. 3.1 below to determine the strength of the magnetic field. At low temperatures, we know that the positions of the $n t h$ harmonic for a given magnetic field $(B)$ and viewing angle $(\theta)$ is given by the following equation:

$$
\lambda_{n}=\frac{10710}{n}\left(\frac{10^{8} \mathrm{G}}{B}\right) \sin \theta
$$

where $\lambda_{n}$ is the wavelength of the peak of the harmonic and $n$ is the harmonic number. We assumed that $\theta=70^{\circ}$, this corresponds to the mean magnetic field of $\sim 58 \mathrm{MG}$. As is evident from the figure, not all the harmonics can be described by the model. This is because the positions of the harmonics depend on many properties such as the electron temperature, optical depth and the viewing angle $\theta$. Also, it is not possible to fit all the harmonics seen with the single value of the magnetic field.

\subsection{MeerKAT radio results}

Our observations taken with the MeerKAT telescope in imaging mode show a faint source,

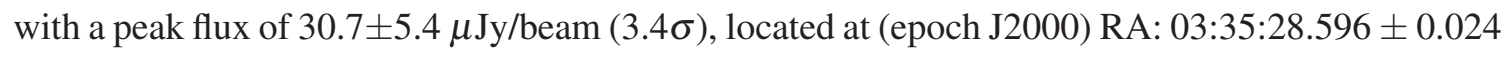
and Dec: $-25: 44: 21.331 \pm 0.344$. The rms noise is estimated to be $9 \mu \mathrm{Jy} / \mathrm{beam}$. The synthesized beam size is $7.45 \times 5.91 \mathrm{arsec}^{2}$ at a position angle of $-35.81^{\circ}$. The position of this source coincides, within the uncertainty given, with the optical coordinates of UZ For - RA: 03:35:28.652 \pm 0.048 and Dec: -25:44:21.766 \pm 0.057 (epoch J2000, Gaia Collaboration 2018).

\section{Discussion and conclusions}

We presented phase-resolved spectroscopy and spectropolarimetry of UZ For. Our averaged spectrum of UZ For is dominated by emission from HeII $4686 \AA$ and the Balmer lines. The spectra show a flat Balmer decrement and this is not consistent with the steep Balmer decrement reported by Allen et al. (1989). We used the spectroscopic results to compute Doppler maps of the strongest emission lines. Our standard and inside-out Doppler tomograms show three emission components: the emission from the irradiated secondary star, emission from the ballistic and magnetic confined stream. These components are more obvious in the inside-out tomogram. In the standard projection it is difficult to distinguish the various emission components seen in the trailed spectra since the ballistic stream and the threading region dominates the emission. Our Doppler map results of the $\mathrm{H} \beta$ line are consistent with those presented by Schwope et al. (1999) for HeII 4686 $\mathrm{A}$. Our modulation amplitude maps show that at least two emission components are flux modulated: the ballistic and the magnetic confined accretion stream, are obviously modulated in both tomograms. The Doppler maps presented here are dominated by emission from the ballistic and magnetic confined stream.

We also presented linear polarization of UZ For and the results shows polarization reaching $\sim 5 \%$ when out of eclipse. A pulse of linear polarization is seen just after the eclipse reaching $\sim 10 \%$ and decreasing gradually before phase 1.1. After that, the polarization is consistent with 5\%. The percentage of linear polarization is consistent with the results presented by Berriman \& Smith (1988). Our circular- photopolarimetry and spectropolarimetry results are consistent with 
each in the sense that we see negative polarization leading to the primary eclipse in both with polarization reaching $-5 \%$ in in former and $-8 \%$ in the latter. Our circular polarization results are not consistent with those reported in literature by Berriman \& Smith (1988) and Ferrario et al. (1989), who both detected positive circular polarization.

The circularly polarized spectra showed three cyclotron harmonics associated with harmonic numbers 4, 3 and 2, respectively. These three features weakens going into the eclipse. When the WD and the accretion spot(s) are emerging from the eclipse, only the strongest dip ( 4500 $\AA$ ) is still visible and there is a possibility of an additional dip below $4000 \AA$ but this appears positive. The additional dip is present in the spectra presented by Ferrario et al. (1989). After the eclipse, the spectrum appears flat and exhibit nonzero polarization in the red. The resulting polarized flux from this cyclotron spectra was modelled using pure cyclotron models with the magnetic field strength of 57 MG. We note that our overlayed model does not fit all the humps well especially in the red part of the spectrum. We attribute this to the contribution from the secondary star amongst other things.

We detected radio emission at the expected position of UZ For using MeerKAT in the L-band centred at $1.28 \mathrm{GHz}$ with a peak flux of $30.7 \pm 5.4 \mu \mathrm{Jy} /$ beam. The reported magnitude of $\mathrm{UZ}$ For around the time of MeerKAT observations by the AAVSO ranges between 16.5-16.1. These magnitudes are consistent with those derived from MeerLICHT observations taken at the same time as the radio observations. Recently, Barrett et al. (2017) detected UZ For in the radio using the VLA at C-band (4-6 GHz) they found it to have a flux density of $315 \pm 101 \mu \mathrm{Jy}$ and showed weak circular polarization reaching percentage of $13 \pm 19$. We have detected UZ For at a flux density ten times fainter that previous recorded and this is accredited to the sensitivity of the MeerKAT telescope. Our results suggest that UZ For is variable in radio wavelengths but the time-scales of this source's variability is not yet known. UZ For lies in one of the MIGHTEE (Jarvis et al., 2016) fields fields and we therefore will continue monitoring it in optical and radio wavelengths.

\section{Acknowledgements}

The spectroscopic observations reported in this paper were obtained with the Southern African Large Telescope (SALT) in the facilities of the SAAO in Sutherland. We thank the staff at the South African Radio Astronomy Observatory (SARAO) for scheduling these observations. The MeerKAT telescope is operated by the South African Radio Astronomy Observatory, which is a facility of the National Research Foundation, an agency of the Department of Science and Innovation. We acknowledge use of the Inter-University Institute for Data Intensive Astronomy (IDIA) data intensive research cloud for data processing. IDIA is a South African university partnership involving the University of Cape Town, the University of Pretoria and the University of the Western Cape.

\section{DISCUSSION}

MARK KENNEDY: Do you know the distance to UZ For?

Answer: Yes, it is about $240 \mathrm{pc}$. 
DAVID BUCKLEY: Have you created a residual trailed spectrum (i.e. observed minus calculated/predicted) which I imagine might be useful in diagnosing the mis-match of observed and model tomograms?

Yes, we have created the residual spectrum for each of the method used; the standard map and the inside-out map, however, they were not shown in the presentation. When we compare the two residuals maps, the one based on the inside-out has the least residuals than the one based on the standard map.

\section{References}

Allen, R. G., Berriman, G., Smith, P. S., \& Schmidt, G. D. 1989, ApJ, 347, 426

Bailey, J. \& Cropper, M. 1991, MNRAS, 253, 27

Baldwin, J. A. \& Stone, R. P. S. 1984, MNRAS, 206, 241

Barrett, P. E., Dieck, C., Beasley, A. J., Singh, K. P., \& Mason, P. A. 2017, AJ, 154, 252

Berriman, G. \& Smith, P. S. 1988, ApJ, 329, L97

Bloemen, S., Groot, P., Woudt, P., et al. 2016, Society of Photo-Optical Instrumentation Engineers (SPIE) Conference Series, Vol. 9906, MeerLICHT and BlackGEM: custom-built telescopes to detect faint optical transients, 990664

Buckley, D. A. H., Burgh, E. B., Cottrell, P. L., et al. 2006, in Proc. SPIE, Vol. 6269, Society of Photo-Optical Instrumentation Engineers (SPIE) Conference Series, 62690A

Burgh, E. B., Nordsieck, K. H., Kobulnicky, H. A., et al. 2003, in Proc. SPIE, Vol. 4841, Instrument Design and Performance for Optical/Infrared Ground-based Telescopes, ed. M. Iye \& A. F. M. Moorwood, 1463-1471

Camilo, F., Scholz, P., Serylak, M., et al. 2018, ApJ, 856, 180

Coppejans, R., Gulbis, A. A. S., Kotze, M. M., et al. 2013, PASP, 125, 976

Crawford, S. M., Still, M., Schellart, P., et al. 2010, in Proc. SPIE, Vol. 7737, Observatory Operations: Strategies, Processes, and Systems III, 773725

Cropper, M. 1990, Space Sci. Rev., 54, 195

Cropper, M., Wu, K., Ramsay, G., \& Kocabiyik, A. 1999, MNRAS, 306, 684

Eastman, J., Siverd, R., \& Gaudi, B. S. 2010, PASP, 122, 935

Euchner, F., Reinsch, K., Jordan, S., Beuermann, K., \& Gänsicke, B. T. 2005, A\&A, 442, 651

Fender, R., Woudt, P. A., Armstrong, R., et al. 2017, arXiv e-prints, arXiv:1711.04132

Ferrario, L., Wickramasinghe, D. T., Bailey, J., Tuohy, I. R., \& Hough, J. H. 1989, ApJ, 337, 832 
Gaia Collaboration. 2018, VizieR Online Data Catalog, I/345

Jarvis, M., Taylor, R., Agudo, I., et al. 2016, in MeerKAT Science: On the Pathway to the SKA, 6

Jonas, J. \& MeerKAT Team. 2016, in Proceedings of MeerKAT Science: On the Pathway to the SKA. 25-27 May, 1

Khangale, Z. N., Potter, S. B., Kotze, E. J., Woudt, P. A., \& Breytenbach, H. 2019, A\&A, 621, A31

Kobulnicky, H. A., Nordsieck, K. H., Burgh, E. B., et al. 2003, in Proc. SPIE, Vol. 4841, Instrument Design and Performance for Optical/Infrared Ground-based Telescopes, ed. M. Iye \& A. F. M. Moorwood, 1634-1644

Kotze, E. J., Potter, S. B., \& McBride, V. A. 2015, A\&A, 579, A77

McMullin, J. P., Waters, B., Schiebel, D., Young, W., \& Golap, K. 2007, in Astronomical Society of the Pacific Conference Series, Vol. 376, Astronomical Data Analysis Software and Systems XVI, ed. R. A. Shaw, F. Hill, \& D. J. Bell, 127

Meggitt, S. M. A. \& Wickramasinghe, D. T. 1982, MNRAS, 198, 71

Nordsieck, K. H., Jaehnig, K. P., Burgh, E. B., et al. 2003, in Society of Photo-Optical Instrumentation Engineers (SPIE) Conference Series, Vol. 4843, Proc. SPIE, ed. S. Fineschi, 170-179

Offringa, A. R., van de Gronde, J. J., \& Roerdink, J. B. T. M. 2012, A\&A, 539, A95

Perryman, M. A. C., Cropper, M., Ramsay, G., et al. 2001, MNRAS, 324, 899

Potter, S., Ramsay, G., Wu, K., \& Cropper, M. 2002, in Astronomical Society of the Pacific Conference Series, Vol. 261, The Physics of Cataclysmic Variables and Related Objects, ed. B. T. Gänsicke, K. Beuermann, \& K. Reinsch, 165

Potter, S. B. 1998, PhD thesis, Mullard Space Science Laboratory, University College London, Holmbury St. Mary, Dorking, Surrey RH5 6NT, UK

Potter, S. B., Buckley, D. A. H., O’Donoghue, D., et al. 2010, MNRAS, 402, 1161

Potter, S. B., Nordsieck, K., Romero-Colmenero, E., et al. 2016, in Society of Photo-Optical Instrumentation Engineers (SPIE) Conference Series, Vol. 9908, Proc. SPIE, 99082K

Schmidt, G. D., Szkody, P., Smith, P. S., et al. 1996, ApJ, 473, 483

Schwope, A. D., Schwarz, R., Staude, A., et al. 1999, in Astronomical Society of the Pacific Conference Series, Vol. 157, Annapolis Workshop on Magnetic Cataclysmic Variables, ed. C. Hellier \& K. Mukai, 71

Tapia, S. 1977, ApJ, 212, L125

Tasse, C., Hugo, B., Mirmont, M., et al. 2018, A\&A, 611, A87

Warner, B. 1995, Cambridge Astrophysics Series, 28 
Wickramasinghe, D. T. \& Meggitt, S. M. A. 1982, MNRAS, 198, 975

-. 1985, MNRAS, 214, 605 